\title{
Ladder operator formalisms and generally deformed oscillator algebraic structures of quantum states in Fock space
}

\author{
Xiaoguang Wang* \\ CCAST(World Laboratory),P.O.Box 8730, Beijing 100080 \\ and Laboratory of Optical Physics, Institute of Physics, \\ Chinese Academy of Sciences, Beijing 100080, People's Republic of China
}

(December 24, 2017)

\begin{abstract}
We show that various kinds of one-photon quantum states studied in the field of quantum optics admit ladder operator formalisms and have the generally deformed oscillator algebraic structure. The two-photon case is also considered. We obtain the ladder operator formalisms of two general states defined in the even/odd Fock space. The two-photon states may also have a generally deformed oscillator algebraic structure. Some interesting examples of one-photon and two-photon quantum states are given.
\end{abstract}

Typeset using REVTEX

*email:xyw@aphy.iphy.ac.cn 


\section{INTRODUCTION}

The interesting quantum states of the radiation field such as coherent states(CSs)t, squeezed states $(\mathrm{SSs})$ 目, binomial states $(\mathrm{BSs})$ 色 and negative binomial states $(\mathrm{NBSs})$ 目 have been studied in detail in the literature. The well known CS has many applications in both quantum optics and condensed matter physics. The CS is the eigenvector of the boson annihilation operator, while the SS is the eigenvector of a linear combination of the boson annihilation and creation operators. The BS interpolates between two fundamental states, the CS and the Fock state, and reduces to them in two different limits3. It partakes the properties of the CS and the Fock state. The photon number distribution of the BS is binomial distribution in probability theory, while the photon number distribution of the NBS is negative binomial distribution. The NBS can also reduce to the $\mathrm{CS}$ in a certain limit.

The CS has the Heisenberg-Weyl algebraic structure while the SS has the su(1,1) Lie algebraic structure. It is found that the BS and the NBS also have Lie algebraic structures. The algebra involved in the BS $\mathrm{E}^{\mathrm{E}}$ is the $\mathrm{su}(2)$ Lie algebra via HolsteinPrimakoff realization 6 and that involved in the NBS ${ }^{6}$ is the $\mathrm{su}(1,1)$ Lie algebra via Holstein-Promakoff realization. It is well known that the CS and SS can be written as the displacement operator formalisms. The BS and NBS also admit displacement operator formalisms. As a generalization of the BS and a generalization of both the BS and the NBS, the hypergeometric state(HGS) 8 and Pólya state(PS) 9 are introduced by $\mathrm{Fu}$ et al, respectively. The HGS reduce to BS in a certain limit and the NHGS reduces to the BS and the NBS in two different limits. The photon number distribution of the HGS is the hypergeometric distribution in probability theory and that of the NHGS is the Pólya distribution. The algebraic structure of the HGS and PS are the well investigated generally deformed oscillator (GDO) algebra 10 . Recently, Roy et al. and Fan et al. also introduce two kinds of quantum states which are claimed to be BS-NBS intermediate states1 12 , 2 , but we have shown that these two kinds of states are identical to the PS\$1 13. 
We see that every quantum state mentioned above has its own algebraic structure. Once natural question is: what is the algebraic structure for a general quantum state? Usually we often study three classes of quantum states. One is the state with linear combination of first finite Fock states, another is the state without the first finite Fock states, and the last one is the state with all Fock states. In this paper, we will investigate these three classes of general states and find their algebraic structures and ladder operator formalisms. Both one-photon and two-photon quantum states are considered.

The GDO algebra first appeared in Heisenberg's theory of nonlinear spinor dynamics 10 . Many physical systems are found to posess the GDO algebra symmetry 14 . The GDO albebra is an associate algebra over the complex number field $C$ with generators $A^{+}, A, \mathcal{N}$ and the unit 1 satisfying

$$
\left[\mathcal{N}, A^{+}\right]=A^{+},[\mathcal{N}, A]=-A, A A^{+}=F(\mathcal{N}+1), A^{+} A=F(\mathcal{N})
$$

where the Hermitian non-negative function $F$ is called the structure function, which should satisfy the condition $F(0)=0$ in order to have Fock representation. It will be shown that many quantum states bear GDO algebraic structure.

\section{ONE-PHOTON QUANTUM STATES}

\section{A. Quantum states as a linear combination of first finite Fock states}

We first consider the state $|x, M\rangle$ defined as a linear combination of first finite Fock states in an $(M+1)$-dimensional subspace of the Fock space

$$
|x, M\rangle=\sum_{n=0}^{M} C(n, x, M)|n\rangle,
$$

where $x$ denotes parameters of the state, $M$ is a non-negative integer and $|n\rangle$ is the usual Fock state. We assume the coefficients in this paper are all non-zero. One typical example of the state $|x, M\rangle$ is the BS. Next we want to find the ladder operator formalism of the general state $|x, M\rangle$. 
Let the operator $f(\hat{N}) a$ act on the state and we get

$$
f(\hat{N}) a|x, M\rangle=\sum_{n=0}^{M-1} f(n) C(n+1, x, M) \sqrt{n+1}|n\rangle,
$$

where $f(\hat{N})$ is a nonlinear function of the operator $\hat{N}=a^{+} a$. Here $a^{+}$and $a$ are the creation and annihilation operators of the radiation field, respectively. If we choose

$$
f(\hat{N})=\frac{C(\hat{N}, x, M-1)}{\sqrt{\hat{N}+1} C(\hat{N}+1, x, M)},
$$

Eq.(3) becomes

$$
f(\hat{N}) a|x, M\rangle=|x, M-1\rangle .
$$

The operator $f(\hat{N}) a$ transforms the state $|x, M\rangle$ to the state $|x, M-1\rangle$. We find another operator $g(\hat{N}) \sqrt{M-\hat{N}}$ which can make this transformation. It is easy to evaluate that

$$
g(\hat{N}) \sqrt{M-\hat{N}}|x, M\rangle=|x, M-1\rangle
$$

where

$$
g(\hat{N})=\frac{C(\hat{N}, x, M-1)}{\sqrt{M-\hat{N}} C(\hat{N}, x, M)} .
$$

The operator $\sqrt{M-\hat{N}}$ removes the number state $|M\rangle$ from the state $|x, M\rangle$ and the operator $g(\hat{N})$ makes the removed state be the state $|x, M-1\rangle$. Note that the operator $g(\hat{N}) \sqrt{M-\hat{N}}$ is not equal to the operator $C(\hat{N}, x, M-1) / C(\hat{N}, x, M)$ since the operator $1 / \sqrt{M-\hat{N}}$ is defined in the $M$-dimensional subspace instead of $(M+$ 1)-dimensional subspace.

Combining Eqs.(5) and (6) leads to

$$
f(\hat{N}) a|x, M\rangle=g(\hat{N}) \sqrt{M-\hat{N}}|x, M\rangle
$$

Substituting Eqs.(4) and (7) into the above equation, we obtain the ladder operator formalism of the state $|x, M\rangle$ as

$$
\left[\hat{N}+\frac{(M-\hat{N}) C(\hat{N}, x, M)}{\sqrt{\hat{N}+1} C(\hat{N}+1, x, M)} a\right]|x, M\rangle=M|x, M\rangle .
$$


Now let us examine the algebraic structure involved in the above equation. Define $\mathcal{A}$ as an associate algebra with generators

$$
\hat{N}, A_{M}^{-}=\frac{(M-\hat{N}) C(\hat{N}, x, M)}{\sqrt{\hat{N}+1} C(\hat{N}+1, x, M)} a, A_{M}^{+}=\left(A_{M}^{-}\right)^{\dagger} .
$$

Then it is easy to verify that these operators satisfy the commutation relations

$$
\left[\hat{N}, A_{M}^{ \pm}\right]= \pm A_{M}^{ \pm}, A_{M}^{+} A_{M}^{-}=F(\hat{N}), A_{M}^{-} A_{M}^{+}=F(\hat{N}+1)
$$

where the function

$$
F(\hat{N})=(M-\hat{N}+1)^{2} \frac{C^{2}(\hat{N}-1, x, M)}{C^{2}(\hat{N}, x, M)} .
$$

This algebra $\mathcal{A}$ is nothing but the GDO algebra with the structure function $F(\hat{N})$.

In terms of the generators of the algebra $\mathcal{A}$, Eq.(8) can be rewritten as

$$
\left(\hat{N}+A_{M}^{-}\right)|x, M\rangle=M|x, M\rangle .
$$

Below we study several interesting special cases of the state $|x, M\rangle$.

1. The BS is defined as

$$
|\eta, M\rangle=\sum_{n=0}^{M}\left[\left(\begin{array}{c}
M \\
n
\end{array}\right) \eta^{n}(1-\eta)^{M-n}\right]^{1 / 2}|n\rangle .
$$

Here $\eta$ is a real parameter satisfying $0<\eta<1$. From Eq.(9) and (14), we get the following ladder operator formalism for the BS

$$
[\hat{N}+\sqrt{(1-\eta) / \eta} \sqrt{(M-\hat{N})} a]|\eta, M\rangle_{B S}=M|\eta, M\rangle_{B S}
$$

which is identical to that obtained in Ref.5.

2. As a one-parameter generalization of the BS, the HGS is given by 8

$$
|L, M, \eta\rangle_{H G S}=\sum_{n=0}^{M}\left[\left(\begin{array}{c}
L \eta \\
n
\end{array}\right)\left(\begin{array}{c}
L \bar{\eta} \\
M-n
\end{array}\right)\right]^{1 / 2}\left(\begin{array}{c}
L \\
M
\end{array}\right)^{-1 / 2}|n\rangle,
$$

where $\bar{\eta}=1-\eta, L$ is a real number satisfying $L \geq \max \left\{M \eta^{-1}, M \bar{\eta}^{-1}\right\}$, and

$$
\left(\begin{array}{l}
x \\
n
\end{array}\right)=\frac{x(x-1) \ldots(x-n+1)}{n !},\left(\begin{array}{l}
x \\
0
\end{array}\right) \equiv 1 .
$$


From the above equation and Eq.(9), we obtain

$$
\left[\hat{N}+\left(\frac{L \bar{\eta}-M+\hat{N}+1}{L \eta-\hat{N}}\right)^{1 / 2} \sqrt{(M-\hat{N})} a\right]|L, M, \eta\rangle_{H G S}=M|L, M, \eta\rangle_{H G S}
$$

which is the ladder operator formalism of the HGS.

3.As a BS-NBS intermediate state, the PS is introduced as

$$
|\eta, \gamma, M\rangle_{P S}=\sum_{n=0}^{M} P_{n}^{M}(\gamma, \eta)|n\rangle
$$

where

$$
\begin{aligned}
P_{n}^{M}(\gamma, \eta)= & \left(\begin{array}{c}
M \\
n
\end{array}\right)^{1 / 2}\left\{\prod_{k=1}^{n}[\eta+(k-1) \gamma]\right\}^{1 / 2}\left\{\prod_{k=1}^{M-n}[\bar{\eta}+(k-1) \gamma]\right\}^{1 / 2} \\
& \left\{\prod_{k=1}^{M}[1+(k-1) \gamma]\right\}^{-1 / 2}
\end{aligned}
$$

and $\gamma>0$ is a real constant.

The ladder operator formalism of the PS is obtained as

$$
\left[\hat{N}+\left(\frac{\bar{\eta}+(M+\hat{N}-1) \gamma}{\eta+\hat{N} \gamma}\right)^{1 / 2} \sqrt{(M-\hat{N})} a\right]|\eta, \gamma, M\rangle_{P S}=M|\eta, \gamma, M\rangle_{P S}
$$

4. As a reference state to detect the phase of a quantum state, Barnett and Pegg introduced a reciprocal binomial state(RBS) 1 国

$$
|\theta, M\rangle_{R B S}=\left(1 / \sum_{n=0}^{M}\left(\begin{array}{c}
M \\
n
\end{array}\right)^{-1}\right) \sum_{n=0}^{M}\left(\begin{array}{c}
M \\
n
\end{array}\right)^{-1 / 2} \exp (i n \theta)|n\rangle .
$$

The ladder operator formalism is directly obtained as

$$
\left[\hat{N}+\frac{M-\hat{N}}{\hat{N}+1} \exp (-i \theta) \sqrt{(M-\hat{N})} a\right]|\theta, M\rangle_{R B S}=M|\theta, M\rangle_{R B S} .
$$

5.Pegg and Barnett 16 defined the Hermitian phase operator on a finite-dimensional state space, which makes it possible to study the phase properties of quantum states of a single mode of electromagnetic field. The Pegg-Barnett phase states (PBPS) $\left|\theta_{m}\right\rangle$ can be defined as

$$
\left|\theta_{m}, M\right\rangle_{P B P S}=\frac{1}{(M+1)^{1 / 2}} \sum_{n=0}^{M} \exp \left(i n \theta_{m}\right)|n\rangle
$$


The phase states in Eq.(24) form an orthonormal set provided that we have

$$
\theta_{m}=\theta_{0}+2 \pi m /(s+1), m=0,1, \ldots, s,
$$

where $\theta_{0}$ is an arbitrary reference phase.

We give the ladder operator formalism of the PBPS as

$$
\left[\hat{N}+\frac{M-\hat{N}}{\sqrt{\hat{N}+1}} \exp \left(-i \theta_{m}\right) a\right]\left|\theta_{m}, M\right\rangle_{P B P S}=M\left|\theta_{m}, M\right\rangle_{P B P S} .
$$

6. The generalized geometric state (GGS) is defined as 17

$$
|Y, M\rangle_{G G S}=\left(\frac{1-|Y|}{1-|Y|^{M+1}}\right)^{1 / 2} \sum_{n=0}^{M} Y^{n / 2}|n\rangle
$$

where $Y$ is a complex parameter.

The ladder operator formalism of the GGS is directly given as

$$
\left[\hat{N}+\frac{M-\hat{N}}{\sqrt{Y} \sqrt{\hat{N}+1}} a\right]|Y, M\rangle_{G G S}=M|Y, M\rangle_{G G S} .
$$

We also give the corresponding structure functions of the above six quantum states, the BS, HGS, PS, RBS, PBPS and GGS as follows:

$$
\begin{aligned}
F_{B S}(N) & =(M-N+1)^{3} \frac{1-\eta}{\eta}, \\
F_{H G S}(N) & =(M-N+1)^{3} \frac{L(1-\eta)-M+N}{L \eta-N+1}, \\
F_{P S}(N) & =(M-N+1)^{3} \frac{(1-\eta)+(M+N-2) \gamma}{\eta+(N-1) \gamma}, \\
F_{R B S}(N) & =\exp (-2 i \theta)(M-N+1)^{5} / N^{2}, \\
F_{P B P S}(N) & =\exp \left(-2 i \theta_{m}\right)(M-N+1)^{4} / N, \\
F_{G G S}(N) & =(M-N+1)^{4} /(Y N) .
\end{aligned}
$$

\section{B. Quantum states without first finite Fock states}

We further investigate the state $|x, M\rangle^{-}$defined as

$$
|x, M\rangle^{-}=\sum_{n=M}^{\infty} D(n, x, M)|n\rangle .
$$


This state has no first finite Fock states. One type of this state is the so-called photon-added quantum state 18

$$
|\psi, M\rangle=N_{M} a^{\dagger M}|\psi\rangle
$$

where $|\psi\rangle$ may be an arbitrary quantum state

$$
|\psi\rangle=\sum_{n=0}^{\infty} C(n, x)|n\rangle
$$

and $N_{M}$ is a normalization constant. For the first time, the photon-added states were introduced by Agarwal and Tara as photon-added coherent states(PACSs) 1 .

Let the operators $f(\hat{N}) a^{\dagger}$ and $g(\hat{N}) \sqrt{\hat{N}-M}$ act on the state $|x, M\rangle^{-}$, which leads to

$$
\begin{aligned}
f(\hat{N}) a^{\dagger}|x, M\rangle^{-} & =|x, M+1\rangle^{-}, \\
g(\hat{N}) \sqrt{\hat{N}-M}|x, M\rangle^{-} & =|x, M+1\rangle^{-},
\end{aligned}
$$

where

$$
\begin{aligned}
f(\hat{N}) & =\frac{D(\hat{N}, x, M+1)}{\sqrt{\hat{N}} D(\hat{N}-1, x, M)}, \\
g(\hat{N}) & =\frac{D(\hat{N}, x, M+1)}{\sqrt{\hat{N}-M} D(\hat{N}, x, M)} .
\end{aligned}
$$

The operators used here, $f(\hat{N}) a^{\dagger}$ and $g(\hat{N}) \sqrt{\hat{N}-M}$, are different from those in deriving the ladder operator formalism of the state $|x, M\rangle$. By applying the two operators on the state $|x, M\rangle^{-}$, we can transform the state $|x, M\rangle^{-}$to $|x, M+1\rangle^{-}$.

From Eqs.(33)-(36), we obtain

$$
\left[\hat{N}-\frac{(\hat{N}-M) D(\hat{N}, x, M)}{\sqrt{\hat{N}} D(\hat{N}-1, x, M)} a^{\dagger}\right]|x, M\rangle^{-}=M|x, M\rangle^{-} .
$$

This is the ladder operator formalism of the state $|x, M\rangle^{-}$in terms of the operators $\hat{N}$ and $a^{\dagger}$. By multiplying the annihilation operator $a$ on the above equation from left, it can be written in terms of the operators $\hat{N}$ and $a$

$$
(\hat{N}+1-M) a|x, M\rangle^{-}=\frac{(\hat{N}+1-M) \sqrt{\hat{N}+1} D(\hat{N}+1, x, M)}{D(\hat{N}, x, M)}|x, M\rangle^{-}
$$


As a special case of the state $|x, M\rangle^{-}$, the photon-added state(Eq.(31)) can be expanded as

$$
|\psi, M\rangle=N_{M} \sum_{n=M}^{\infty} C(n-M, x)[n ! /(n-M) !]^{1 / 2}|n\rangle .
$$

From the above equation and Eq.(37), we obtain the ladder operator formalism of the photon-added state as

$$
\left[\hat{N}-\frac{C(\hat{N}-M, x)}{C(\hat{N}-M-1, x)} \sqrt{\hat{N}-M} a^{\dagger}\right]|\psi, M\rangle=M|\psi, M\rangle .
$$

The above equation can be written in terms of the operators $\hat{N}$ and $a$,

$$
\left.(\hat{N}+1-M) a|\psi, M\rangle=\frac{C(\hat{N}+1-M, x)}{C(\hat{N}-M, x)} \sqrt{\hat{N}+1-M}(\hat{N}+1)\right]|\psi, M\rangle .
$$

The algebra involved in the state $|x, M\rangle^{-}$is also the GDO algebra $\mathcal{B}$, with the generators

$$
\hat{N}, B_{M}^{+}=\frac{(\hat{N}-M) D(\hat{N}, x, M)}{\sqrt{\hat{N}} D(\hat{N}-1, x, M)} a^{\dagger}, B_{M}^{-}=\left(B_{M}^{+}\right)^{\dagger} .
$$

These satisfy the commutation relations

$$
\left[\hat{N}, B_{M}^{ \pm}\right]= \pm B_{M}^{ \pm}, B_{M}^{+} B_{M}^{-}=G(\hat{N}), B_{M}^{-} B_{M}^{+}=G(\hat{N}+1)
$$

with the structure function

$$
G(\hat{N})=(\hat{N}-M)^{2} \frac{D^{2}(\hat{N}, x, M)}{D^{2}(\hat{N}-1, x, M)} .
$$

In terms of the generators of the algebra $\mathcal{B}$, Eq.(37) becomes

$$
\left[\hat{N}-B_{M}^{+}\right]|x, M\rangle^{-}=M|x, M\rangle^{-}
$$

As an example, we derive the ladder operator formalism for the PACS. The CS expanded in Fock space is

$$
|\psi\rangle_{C S}=\exp \left(-|\alpha|^{2} / 2\right) \sum_{n=0}^{\infty} \frac{\alpha^{n}}{\sqrt{n !}}|n\rangle
$$

where $\alpha$ is a complex number. Using the coefficients of the CS and Eq.(41), we find the ladder operator formalism for the PACS to be

$$
(\hat{N}+1-M) a|\alpha, M\rangle=\alpha(\hat{N}+1)|\alpha, M\rangle .
$$


Since the operator $1 /(\hat{N}+1)$ is non-zero in the whole Fock space, we get

$$
[1-M /(\hat{N}+1)] a|\alpha, M\rangle=\alpha|\alpha, M\rangle
$$

According to the definition of the nonlinear coherent states (NLCSs)

$$
f(\hat{N}) a|\alpha\rangle_{N L C S}=\alpha|\alpha\rangle_{N L C S}
$$

so the PACS is an NLCS as discussed by Sivakumane 20 . Here $f(\hat{N})$ is a nonlinear function of $\hat{N}$.

As another example, we consider a new definition of NBS(NNBS)21 recently introduced by Barnett:

$$
|\eta, M\rangle_{N N B S}=\sum_{n=M}^{\infty}\left[\left(\begin{array}{c}
n \\
M
\end{array}\right) \eta^{M+1}(1-\eta)^{n-M}\right]^{1 / 2}|n\rangle,
$$

It is found that the NNBS and the BS have similar properties if the roles of the creation operator $a^{\dagger}$ and annihilation operator $a$ are interchanged.

Form Eq.(38), the ladder operator formalism of the NNBS is obtained as

$$
[\sqrt{\hat{N}+1-M} /(\hat{N}+1)] a|\eta, M\rangle_{N N B S}=\sqrt{1-\eta}|\eta, M\rangle_{N N B S}
$$

As seen from the above equation, the NNBS is an NLCS with the nonlinear function $f(\hat{N})=\sqrt{\hat{N}+1-M} /(\hat{N}+1)$. Eq.(51) was obtained in one of our previous papers2

\section{General quantum states}

By an analogous method to that used in obtaining Eq.(37), we obtain the ladder operator formalism of the general state $|\psi\rangle$ (Eq.(32)) as

$$
\left[\hat{N}-\frac{C(\hat{N}, x)}{C(\hat{N}-1, x)} \sqrt{\hat{N}} a^{\dagger}\right]|\psi\rangle=0 .
$$

Multiplying both sides of the above equation by $a$ from the left, we get another form of the ladder operator formalism of the state $|\psi\rangle$,

$$
a|\psi\rangle=\frac{C(\hat{N}+1, x)}{C(\hat{N}, x)} \sqrt{\hat{N}+1}|\psi\rangle .
$$


The algebra involved in the state $|\psi\rangle$ is also the GDO algebra with generators

$$
\hat{N},[C(\hat{N}, x) / C(\hat{N}-1, x)] \sqrt{\hat{N}} a^{\dagger}, a \sqrt{\hat{N}} C(\hat{N}, x) / C(\hat{N}-1, x) .
$$

The corresponding structure function is $\hat{N}^{2} C^{2}(\hat{N}, x) / C^{2}(\hat{N}-1, x)$.

Using the coefficients of the CS (Eq.(46)), we obtain the well-known ladder operator formalism of the CS from Eq.(53):

$$
a|\alpha\rangle_{C S}=\alpha|\alpha\rangle_{C S}
$$

This equation can be naturally obtained by letting $M=0$ in Eq.(48).

Now we consider the geometric state(GS) which is defined as 23

$$
|\eta\rangle_{G S}=\eta^{1 / 2} \sum_{n=0}^{\infty}(1-\eta)^{n / 2}|n\rangle
$$

This is also called the Susskind-Glogower phase state, phase eigenstate,and coherent phase state 23 in the literature. From Eq.(53), we get the ladder operator formalism of the GS

$$
a|\eta\rangle_{G S}=\sqrt{1-\eta} \sqrt{N+1}|\eta\rangle_{G S}
$$

Since the operator $\sqrt{N+1}$ is not zero in the whole Fock space, we obtain

$$
a / \sqrt{N+1}|\eta\rangle_{G S}=\sqrt{1-\eta}|\eta\rangle_{G S}
$$

This equation shows that the GS is an NLCS with the nonlinear function $1 / \sqrt{N+1}$. By setting $M=0$ in Eq.(51), Eq.(51) reduces to Eq.(58). This fact is easily understood since the NNBS can be viewed as photon-added GS22.

Further we study another example, the NBS, which is defined ast

$$
|\eta, M\rangle_{N B S}=\sum_{n=0}^{\infty}(1-\eta)^{M / 2}\left(\begin{array}{c}
M+n-1 \\
n
\end{array}\right)^{1 / 2} \eta^{n / 2}|n\rangle .
$$

From Eq.(53), we get

$$
\frac{1}{\sqrt{M+\hat{N}}} a|\eta, M\rangle_{N B S}=\eta^{1 / 2}|\eta, M\rangle_{N B S}
$$


which shows that the NBS is an NLCS with the nonlinear function $f(\hat{N})=$ $1 / \sqrt{M+\hat{N}}$. This result is identical to that obtained by us before 2 .

It is interesting to investigate the Kerr state (KS) which is defined as 25

$$
|\alpha, \theta\rangle_{K S}=\exp \left(-|\alpha|^{2} / 2\right) \sum_{n=0}^{\infty} \frac{\alpha^{n} \exp [-i \theta n(n-1)]}{\sqrt{n !}}|n\rangle
$$

From Eq.(53), the ladder operator formalism of the KS is obtained as

$$
\exp (-2 i \hat{N} \theta) a|\alpha, \theta\rangle_{K S}=\alpha|\alpha, \theta\rangle_{K S}
$$

When $\theta=0$, Eq.(62) naturally reduce to Eq.(55). Eq.(62) show that the KS is an NLCS with the nonlinear function $f(\hat{N})=\exp (-2 i \hat{N} \theta)$.

Here we give a more general form of Eq.(9) as

$$
(\sqrt{\eta} \hat{N}+\sqrt{1-\eta} f(\hat{N}) a)|\eta, \alpha\rangle=\alpha|\eta, \alpha\rangle
$$

where the parameter $0<\eta<1$ is introduced as discussed in Ref. 27. The parameter $\alpha$ is the eigenvalue to be determined. According to the procedure same as that by $\mathrm{Fu}$ et al.27, we can obtain a new type of quantum state, i.e., the intermediate numbernonlinear coherent states. If we choose $f(\hat{N})=\exp (-2 i \hat{N} \theta)$, we obtain the interesting intermediate number-Kerr state. The detailed discussion will be given elsewhere.

All the states discussed in this section are of the one-photon type. Two-photon quantum states will be studied in the next section.

\section{TWO-PHOTON QUANTUM STATES}

The representative two-photon states are the squeezed vacuum states(SVSs) (defined below) and even/odd coherent states(ECS/OCS)2 These states are defined in either even Fock space or odd Fock space.

The squeezed vacuum state is defined as

$$
|\xi\rangle_{S V S}=S(\xi)|0\rangle
$$


where $S(\xi)=\exp \left(\xi K_{+}-\xi^{*} K_{-}\right)$is the squeezing operator and $\xi=r \exp (i \theta)$. Here $K_{+}=a^{+2} / 2$ and $K_{-}=a^{2} / 2$. The two operators together with the operator $K_{0}=$ $\hat{N} / 2+1 / 4$ form a su(1,1) Lie algebra.

The representation of $\mathrm{su}(1,1)$ on the usual Fock space is completely reducible and decomposes into a direct sum of the even Fock space $\left(S_{0}\right)$ and odd Fock space $\left(S_{1}\right)^{\text {29 }}$,

$$
\left.S_{j}=\operatorname{span}\{|| n\rangle_{j} \equiv|2 n+j\rangle \mid n=0,1,2, \ldots\right\}, j=0,1 .
$$

Representations on $S_{j}$ can be written as

$$
\begin{aligned}
\left.K_{+} \| n\right\rangle_{j} & =\sqrt{(n+1)(n+j+1 / 2)}|| n+1\rangle_{j}, \\
\left.K_{-} \| n\right\rangle_{j} & =\sqrt{(n)(n+j-1 / 2)}|| n-1\rangle_{j}, \\
\left.K_{0} \| n\right\rangle_{j} & =(n+j / 2+1 / 4)|| n\rangle_{j} .
\end{aligned}
$$

The Bargmann index $k=1 / 4(3 / 4)$ for even(odd) Fock space.

It is easily seen that the SQS is defined in the even Fock space. To obtain the expansion of the SQS in the even Fock space, we use the decomposed form of the squeezing operator

$$
S(\xi)=\exp \left(e^{i \theta} \tanh r a^{+2} / 2\right)(\cosh r)^{-\left(a^{+} a+1 / 2\right)} \exp \left(-e^{-i \theta} \tanh r a^{2} / 2\right) .
$$

From the above equation and Eq.(63), we obtain the expansion as

$$
\left.|\xi\rangle_{S V S}=(\cosh r)^{-1 / 2} \sum_{n=0}^{\infty} \sqrt{(2 n) !}\left[e^{i \theta} \tanh r / 2\right]^{n} / n !|| n\right\rangle_{0} .
$$

Now we consider two general states in even/odd Fock space

$$
\begin{aligned}
|x\rangle_{e} & \left.=\sum_{n=0}^{\infty} C_{e}(n, x)|| n\right\rangle_{0}, \\
|x\rangle_{o} & \left.=\sum_{n=0}^{\infty} C_{o}(n, x)|| n\right\rangle_{1} .
\end{aligned}
$$

For convenience, we introduce the number operators $\hat{N}_{0}, \hat{N}_{1}$ defined by 


$$
\begin{aligned}
& \left.\left.\hat{N}_{0}=K_{0}-1 / 4, \hat{N}_{0} \| n\right\rangle_{0}=n \| n\right\rangle_{0}, \\
& \left.\left.\hat{N}_{1}=K_{0}-3 / 4, \hat{N}_{1} \| n\right\rangle_{1}=n \| n\right\rangle_{1} .
\end{aligned}
$$

Using the same method as that used in deriving Eq.(37), we obtain the ladder operator formalisms of the states $|x\rangle_{e / o}$,

$$
\begin{gathered}
\left.\left[\hat{N}_{0}-\frac{\sqrt{\hat{N}_{0}} C_{e}\left(\hat{N}_{0}, x\right)}{C_{e}\left(\hat{N}_{0}-1, x\right) \sqrt{\hat{N}_{0}-1 / 2}} \frac{a^{+2}}{2}\right] x\right\rangle_{e}=0, \\
\left.\left[\hat{N}_{1}-\frac{\sqrt{\hat{N}_{1}} D_{o}\left(\hat{N}_{1}, x\right)}{D_{o}\left(\hat{N}_{1}-1, x\right) \sqrt{\hat{N}_{1}+1 / 2}} \frac{a^{+2}}{2}\right] x\right\rangle_{o}=0 .
\end{gathered}
$$

The above equation can be written in terms of $a^{2}$ and $N_{j}$,

$$
\begin{aligned}
& \left.\left[\frac{C_{e}\left(\hat{N}_{0}+1, x\right) \sqrt{\hat{N}_{0}+1 / 2}\left(N_{0}+1\right)}{C_{e}\left(N_{0}, x\right)}-\sqrt{\hat{N}_{0}+1} \frac{a^{2}}{2}\right] x\right\rangle_{e}=0, \\
& \left.\left[\frac{C_{o}\left(\hat{N}_{1}+1, x\right) \sqrt{N_{1}+3 / 2}\left(N_{1}+1\right)}{C_{o}\left(N_{1}, x\right)}-\sqrt{\hat{N}_{1}+1} \frac{a^{2}}{2}\right] x\right\rangle_{o}=0 .
\end{aligned}
$$

Substituting the coefficients of the SVS into Eq.(72) leads to

$$
\frac{1}{\hat{N}+1} a^{2}|\xi\rangle_{S V S}=\exp (i \theta) \tanh r|\xi\rangle_{S V S}
$$

We see that the SVS is the two-photon nonlinear coherent state(TPNLCS) which can be defined as 26

$$
f(\hat{N}) a^{2}|\alpha\rangle_{T P N L C S}=\alpha|\alpha\rangle_{T P N L C S}
$$

As another squeezed state, the squeezed first excited state(SFES) is defined as

$$
|\xi\rangle_{S F E S}=S(\xi)|1\rangle
$$

By using Eq.(66), we get the expansion of the state $|\xi\rangle_{S F E S}$

$$
\left.|\xi\rangle_{S F E S}=(\cosh r)^{-3 / 2} \sum_{n=0}^{\infty} \sqrt{(2 n+1) !}\left[e^{i \theta} \tanh r / 2\right]^{n} / n !|| n\right\rangle_{0}
$$


Similar to the derivation of Eq.(76), we obtain

$$
\frac{1}{\hat{N}+2} a^{2}|\xi\rangle_{S V S}=\exp (i \theta) \tanh r|\xi\rangle_{S V S}
$$

The above equation shows that the SFES is a TPNLCS.

Other examples are the even CS(ECS) and odd CS(OCS)28

$$
\begin{aligned}
|\alpha\rangle_{E C S} & \left.=1 / \sqrt{\cosh |\alpha|^{2}} \sum_{n=0}^{\infty} \alpha^{2 n} / \sqrt{(2 n) !}|| n\right\rangle_{0,} . \\
|\alpha\rangle_{O C S} & \left.=1 / \sqrt{\sinh |\alpha|^{2}} \sum_{n=0}^{\infty} \alpha^{2 n+1} / \sqrt{(2 n+1) !}|| n\right\rangle_{1} .
\end{aligned}
$$

The ladder operator formalism can be easily obtained as for Eqs(74) and (75)

$$
\begin{aligned}
& a^{2}|\alpha\rangle_{E C S}=\alpha^{2}|\alpha\rangle_{E C S}, \\
& a^{2}|\alpha\rangle_{O C S}=\alpha^{2}|\alpha\rangle_{O C S} .
\end{aligned}
$$

This is just what we expected.

As seen from Eqs.(72) and (73), we conclude that the algebra involved in the two general two-photon states is the GDO algebra with generators

$$
\hat{N}_{0}, \frac{\sqrt{\hat{N}_{0}} C_{e}\left(\hat{N}_{0}, x\right)}{C_{e}\left(\hat{N}_{0}-1, x\right) \sqrt{\hat{N}_{0}-1 / 2}} \frac{a^{+2}}{2}, \frac{a^{2}}{2} \frac{\sqrt{\hat{N}_{0}} C_{e}\left(\hat{N}_{0}, x\right)}{C_{e}\left(\hat{N}_{0}-1, x\right) \sqrt{\hat{N}_{0}-1 / 2}}
$$

and

$$
\hat{N}_{1}, \frac{\sqrt{\hat{N}_{1}} D_{o}\left(\hat{N}_{1}, x\right)}{D_{o}\left(\hat{N}_{1}-1, x\right) \sqrt{\hat{N}_{1}+1 / 2}} \frac{a^{+2}}{2}, \frac{a^{2}}{2} \frac{\sqrt{\hat{N}_{1}} D_{o}\left(\hat{N}_{1}, x\right)}{D_{o}\left(\hat{N}_{1}-1, x\right) \sqrt{\hat{N}_{1}+1 / 2}} .
$$

The corresponding structure functions are

$$
\begin{aligned}
& F_{e}\left(\hat{N}_{0}\right)=\frac{\hat{N}_{0}^{2} C_{e}^{2}\left(\hat{N}_{0}, x\right)}{C_{e}^{2}\left(\hat{N}_{0}-1, x\right)}, \\
& F_{o}\left(\hat{N}_{1}\right)=\frac{\hat{N}_{1}^{2} D_{o}^{2}\left(\hat{N}_{1}, x\right)}{D_{o}^{2}\left(\hat{N}_{1}-1, x\right)} .
\end{aligned}
$$




\section{CONCLUSIONS}

We have shown that various kinds of states in the field of quantum optics admit ladder operator formalisms and have the GDO algebraic structures. The corresponding structure functions have been obtained. As examples we have given ladder operator formalisms for the BS, RBS, NBS, NNBS, HGS, PS, RBS, PBPS, GS, GGS and KS. We have also considered the two-photon case and get the ladder operator formalisms for two general states defined in the even/odd Fock space. We find that the algebra involved in the two general states is also GDO algebra. The ladder operator formalisms of some special two-photon states, the SVS, SFES, ECS and OCS, have been obtained. The SVSs and SFESs are found to be TPNLCSs. In addition, the method proposed here can be applied to the study of ladder operator formalism and the algebraic structure of quantum states for $\mathrm{su}(1,1)$ and $\mathrm{su}(2)$ Lie algebra.

Some quantum states discussed in the present paper seems only for academic interestes. However with the development of the quantum state engineering technique 30 , these states can be fabricated in principle.

Acknowledgments: The author is grateful for discussions with Professor H. C. $\mathrm{Fu}$ and the help of Professors C. P. Sun, S. H. Pan and G. Z. Yang. This work was partially supported by the National Science Foundation of China with Grant No. 19875008. 


\section{REFERENCES}

${ }^{1}$ Glauber R J 1963 Phys.Rev.Lett. 10 277;

Glauber R J 1963 Phys.Rev. 130 2539;

Glauber R J 1963 Phys.Rev. 131 2766;

Zhang W M, Feng D H and Gilmore R 1990 Rev.Mod.Phys. 62867.

${ }^{2}$ Stoler D 1970 Phys.Rev. D1 3217;

Yuen H P 1976 Phys.Rev. A13 2226;

Loudon R and Knight P L 1987 J.Mod.Optics 34709.

${ }^{3}$ Stoler D, Saleh B E A and Teich M C 1985 Opt.Acta. 32345.

Lee C T, 1985 Phys.Rev. A31 1213;

Dottoli G, Gallardo J and Torre A, 1987 J.Opt.Soc.Am. B2 185;

Barranco A V and Roversi J 1994 Phys.Rev. A50 5233;

Fan H Y and Jing S J, 1994 Phys.Rev. A50 1909;

Joshi A and Puri R R, 1987 J.Mod.Opt. 341421.

${ }^{4}$ Joshi A and Lawande S V , 1989 Opt.Commun. 70 21;

Matsuo K, 1990 Phys.Rev. A41 519;

Joshi A and Lawande S V, 1991 J.Mod.Opt. 38 2009;

Agarwal G S, 1992 Phys.Rev. A45 1787;

${ }^{5}$ Fu H C and Sasaki R, 1996 J.Phys.A:Math.Gen. 295637.

${ }^{6}$ Holstein T and Primakoff H, 1940 Phys.Rev. 581098.

${ }^{7}$ Fu H C and Sasaki R, 1997 J.Phys.Soc.Japan 661989.

${ }^{8}$ Fu H Cand R.Sasaki, 1997 J.Math.Phys. 382154.

${ }^{9}$ Fu H C, 1997 J.Phys.A:Math.Gen. 30 L83.

${ }^{10}$ See, for example, Rampacher H, Stumpf H and Wagner F 1965 Fortschritte Phys. 
13385.

${ }^{11}$ Roy P and Roy B, 1997 J.Phys.A:Math.Gen. 30 L719.

${ }^{12}$ Fan H Y and Liu N L, 1998 Phys.Lett. A250 88.

${ }^{13}$ Wang X G, 2000 J. Opt. B: Quantum and Semiclassical Optics 22.

14 Arik M and Coon D D 1976 J.Math.Phys. 17 524;

Banatsos D and Daskaloyannis C 1993 Phys.Lett. B 307 100;

Fu H C and Sasaki R 1996 J.Phys.A: Math. Gen. 294049.

${ }^{15}$ Barnett S M and Pegg D T 1996 Phys.Rev.Lett. 764148

Moussa M H T, Baseia B 1998 Phys. Lett. A238 223.

${ }^{16}$ Pegg D T and Barnett S M, 1988 Europhys.Lett. 6 483;

Pegg D T and Barnett S M, 1989 Phys.Rev.A 39 1665;

Barnett S M and Barnett D T, 1989 J.Mod.Opt. 36 7;

Pegg D T and Barnett S M, 1997 J.Mod.Opt. 44225.

${ }^{17}$ Batarfi H A, Sebawe Abdalla M, Obada A -S F and Hassan S S, 1995 Phys.Rev.A51 2644

Obada A -S F, Hassan S S, Puri R R and Abdalla M S, 1993 Phys.Rev.A48 3173.

${ }^{18}$ Agarwal G S and Tara K, 1991 Phys.Rev. A43 492.

${ }^{19}$ Matos Filho R L de and Vogel W, 1996 Phys.Rev.A54 4560;

Man'ko V I,Marmo G ,Sudarshan E C G, and Zaccaria F, 1997 Physica Scripta, 55 528;

Man'ko O V,1997 Phys.Lett.A228 29;

Mancini S,1997 Phys.Lett.A233 291;

Roy B,1998 Phys.Lett.A249 25.

20 Sivakumar S 1999 J.Phys.A:Math.Gen. 323441. 
${ }^{21}$ Barnett S M, 1998 J.Mod.Opt. 452201.

${ }^{22}$ Wang X G, Pan S H and Yang G Z, accepted in Euro.Phys.J.D, to appear.

${ }^{23}$ Shapiro J H and Shephard S R, 1991 Phys.Rev.Lett. 62 2377;

Shapiro J H and Shephard S R, 1991 Phys.Rev. A 43 3795;

Lerner E C, Huang H W and Walters G E, 1970 J.Math.Phys. 11 1679;

Hall M J W, 1993 J.Mod.Opt. 40 809;

Obada A -S F, Hassan S S, Puri R R and Abdalla M S 1993 Phys.Rev. A48 3174;

Obada A -S F, Yassin O M and Barnett S M, 1997 J.Mod.Opt. 44149.

${ }^{24}$ Wang X G and Fu H C, 1999 Mod.Phys.Lett.B, 13617.

${ }^{25}$ See, for examples, P.Tombesi and H.P.Yuen, in Coherent and Quantum Optics, Vol.V, P.751(Plenum, New York)(1984).

${ }^{26}$ Sivamukar S 1998 Phys.Lett.A 250257.

${ }^{27}$ Fu H C, Feng Y Q and Solomon A I 2000 J. Phys. A: Math. Gen. 33 2231; Sixdeniers J-M and Penson K A 2000 J. Phys. A: Math. Gen. 332907.

${ }^{28}$ Phoenix S J D 1990 Phys.Rev.A41 5132;

Davidovich L, Brune M, Raimond J M and Harone S, 1996 Phys.Rev. A53 1295;

Buzek V, Vidiella-Barranco A and Knight P L, 1992 Phys.Rev. A45 6570;

Raimond J M, Brune M, and Haroche S 1997 Phys.Rev.Lett. 79 1964;

Agarwal G S, 1999 Phys.Rev. A59 3071;

de Matos Filho and Vogel W 1996 Phys.Rev.Lett. 76 608;

Monroe C, Meekof D M, King B E and Wineland D J 1996 Science 272 1131;

Walmsley I A and Raymer M G 1995 Phys.Rev.A52 681

${ }^{29}$ Fu H C and Sasaki R 1996 Phys.Rev.A53 3836.

${ }^{30}$ Janszky J, Domokos P, Szab S, and Adam P, 1995 Phys. Rev. A 51 4191; Dakna M, Knöll L, and Welsch D -G, 1998 Euro. Phys. J. D 3295. 